\title{
Guiding and splitting Lamb waves in coupled-resonator elastic waveguides
}

\author{
Yan-Feng Wang, ${ }^{1, *}$ Ting-Ting Wang, ${ }^{1}$ Jin-Ping Liu, ${ }^{1}$ Yue-Sheng Wang, ${ }^{1,}{ }^{\dagger}$ and Vincent Laude ${ }^{2}$ \\ ${ }^{1}$ Institute of Engineering Mechanics, Beijing Jiaotong University, 100044 Beijing, China \\ ${ }^{2}$ Institut FEMTO-ST, Université de Bourgogne Franche-Comté, CNRS, 25030 Besançon, France
}

\begin{abstract}
We investigate experimentally Lamb wave propagation in coupled-resonator elastic waveguides (CREWs) formed by a chain of cavities in a two-dimensional phononic crystal slab with cross holes. Wide complete bandgaps, extending from 53 to $88 \mathrm{kHz}$, are first measured in a finite phononic crystal slab sample. A straight waveguide and a wave splitting circuit with $90^{\circ}$ bends are then designed, fabricated and measured. Elastic Lamb waves are excited by a piezoelectric patch attached to one side of the phononic slab and detected using a scanning vibrometer. Strongly confined guiding and splitting at waveguide junctions are clearly observed for several guided waves. Numerical simulations are found to be in excellent agreement with experimental results and allow for the identification of the involved resonant cavity modes. The influence on the dispersion of guided waves of the slab thickness and of the hole length is also investigated. The results have implications for the design of innovative phononic devices with strong confinement and tailorable dispersion.
\end{abstract}

Keywords: Phononic crystal, phononic crystal waveguide, Lamb wave, phononic circuits

\section{INTRODUCTION}

Over the past two decades, phononic crystals $(\mathrm{PCs})^{1}$, i.e. artificial functional composites composed of periodic scatterers embedded in a matrix, have attracted a lot of interest ${ }^{2,3}$. In particular, wave propagation can be fully forbidden if the frequency falls inside a phononic bandgap. The factors influencing bandgaps, such as material $^{4}$ and geometrical ${ }^{5}$ parameters, are now well understood. Furthermore, when the geometrical or material properties of one or several unit cells are locally altered, defect states can appear inside bandgaps. As a result, waves can be guided either along a line of defects ${ }^{6,7}$ or along a linear chain of defect cavities ${ }^{8,9}$. As a general rule, confinement of the guided waves is favored by wider bandgaps.

Recently, there has been a growing interest in harnessing the propagation of Lamb waves in PC slabs ${ }^{10-13}$. $\mathrm{PC}$ slabs are periodic and infinite in two directions, but finite in the third direction and hence do not suffer from leakage ${ }^{14}$. Lamb waves are strongly confined between the free surfaces but can still be manipulated in the periodic plane, making them interesting candidates for the design of phononic circuits and devices. Generally, two kinds of PC slabs have been considered: either flat slabs perforated with holes ${ }^{15}$ or containing solid inclusions ${ }^{16}$, or slabs supporting attached pillars ${ }^{17}$ or mechanical resonators ${ }^{18}$. Phononic bandgaps were reported experimentally in slabs at ultrasonic frequencies $^{16,19}$, up to the $\mathrm{GHz}$ range ${ }^{20,21}$. Bandgaps in $\mathrm{PC}$ slabs can be optimized by adding holes ${ }^{22}$ or by using topology optimization $^{23}$. They can also be enlarged by erecting pillars on the solid regions of a perforated $\operatorname{slab}^{24}$ or by adding pillars on both sides of the $\operatorname{slab}^{25}$.

Much attention has also been paid to defect-based waveguides in PC slabs. Investigations have mainly focused on guiding Lamb waves along linear waveguides ${ }^{26}$. Since Lamb wave are channeled along a line defect, com- plex waveguides can be tailored in the plane at frequencies within a complete bandgap. The propagation of Lamb waves can be changed by $90^{\circ}$ through a polyline sharp waveguide ${ }^{27}$ or at a waveguide bend with slightly perturbed lattice in the bent region ${ }^{28}$. Waveguides with many sharp corners $\left(90^{\circ}\right)$ have also been reported, though with a relatively large width ${ }^{17}$. In contrast to linear waveguides, waveguides based on linear chains of coupled cavities have been shown theoretically to allow simultaneously for very strong wave confinement and for low group velocity transmission ${ }^{8,9}$. In this paper, we present the first experimental demonstration of highly confined propagation of Lamb waves along coupled-resonator elastic waveguides (CREWs). We also show that sharp $90^{\circ}$ bends can be included to form

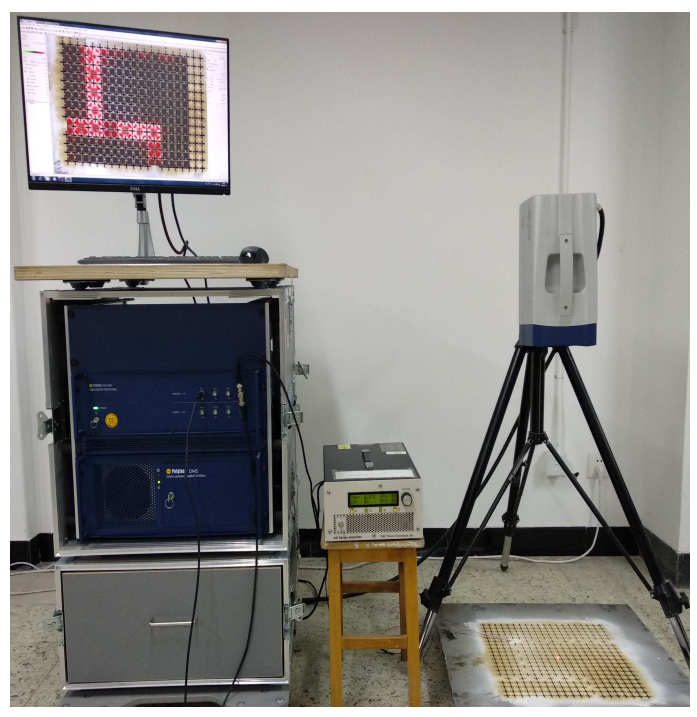

Figure 1: Photograph of the experimental setup illustrating the measurement of vertical displacements of Lamb waves excited in a phononic crystal slab sample. 


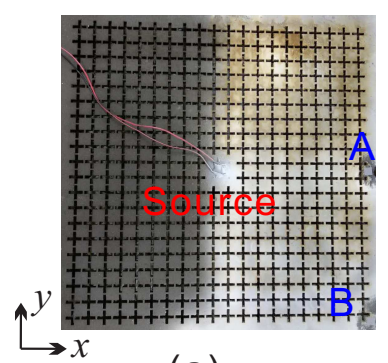

(a)

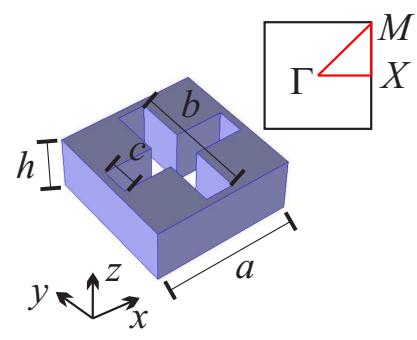

(b)
Figure 2: Phononic crystal slab sample manufactured in a stainless steel plate. (a) The Lamb wave source in the center is a piezoelectric patch. Transmitted waves are measured along the $\Gamma \mathrm{X}$ direction (at point $\mathrm{A}$ ) and the $\Gamma \mathrm{M}$ direction (at point B). (b) The primitive unit cell is schematized, including geometrical dimensions and the first Brillouin zone for the square lattice.

phononic circuits.

\section{EXPERIMENTAL SETUP AND NUMERICAL METHODS}

Fig. 1 presents a photograph of the experimental setup used for measuring Lamb wave propagation in a phononic crystal slab. An asymmetric wave source is formed by sticking a piezoelectric patch to one side of the slab, in order to generate Lamb waves. Two kinds of temporal signals are used. A periodic chirp is used to measure the transmission properties ${ }^{13}$. A harmonic signal with given frequency is further used to measure the distribution of the out-of-plane displacement over the surface of the slab. Displacements are measured using the Polytec PSV-500 scanning vibrometer. Such a technique is often used to detect Lamb waves ${ }^{29,30}$. Transmissions are estimated by detecting out-of-plane displacements around designated points and are averaged over the surface of one unit cell of the phononic crystal.

Numerical simulations are conducted using the finite element method. A primitive unit cell, as sketched in Fig. 2(b), is chosen to determine dispersion relations of the perfect $\mathrm{PC}$ slab. Due to the large impedance ratio between steel and air, air is treated as a vacuum in the numerical model. A supercell technique is used to investigate the dispersion relation and eigenmodes of $\mathrm{CREWs}{ }^{8}$. Traction-free boundary conditions are set on the top and bottom surfaces and inside the holes, and periodic Bloch boundary conditions are applied on the other boundaries. The mesh spacing in the plane of the slab is smaller than $a / 20$, with $a$ the lattice constant. There are 5 elements in the depth of the plate. Second degree polynomial elements of the Lagrange type are used to approximate the displacement vector considered as the unknown. A sparse eigenvalue solver is used to search for eigenfrequencies around $75 \mathrm{kHz}$. Band structures are obtained by letting the wavevector sweep selected directions of the
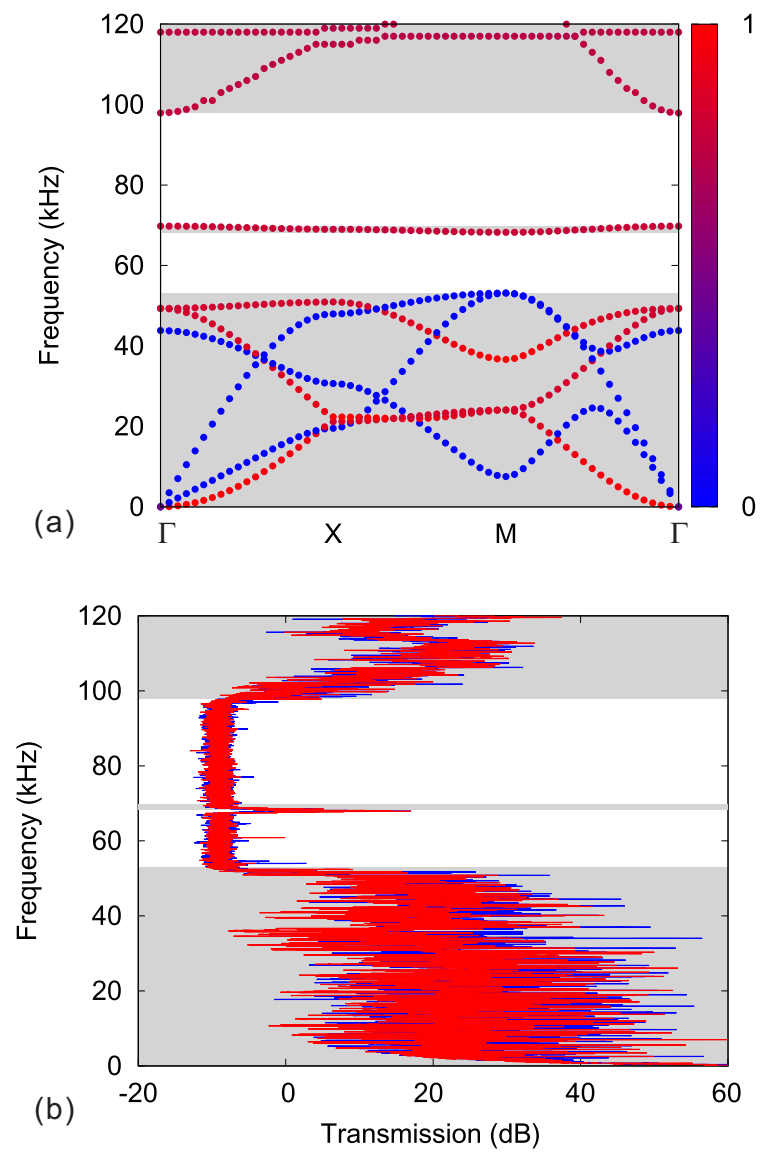

Figure 3: (a) Phononic band structure and (b) experimental transmission of the perfect $\mathrm{PC}$ slab with cross holes $(b / a=0.9, c / a=0.2, h / a=0.4)$. The color scale in panel (a) measures the polarization and varies from in-plane modes (blue) to out-of-plane modes (red). The gray areas indicate the passing frequency ranges. The blue and red lines in panel (b) show measurements along the $\Gamma \mathrm{X}$ and $\Gamma \mathrm{M}$ directions, respectively.

first Brillouin zone. The displacement distribution for selected eigenmodes is obtained by selecting the relevant frequency and wave vector.

Numerical transmissions are further calculated by considering a time-harmonic 3D model of the finite slab. A $z$-polarization displacement wave source with unit amplitude $\left(\left|U_{z 0}\right|=1\right)$ is applied to the central part of the slab. Transmitted displacements are collected at the ends of the waveguide and of the splitter circuit, similarly to the experimental arrangement. The transmission, $T(f)$, is evaluated as the ratio of the vertical displacement amplitude $\left(\left|U_{z}\right|\right)$ integrated over two identical homogeneous parts around the excitation $\left(S_{i}=a^{2}\right)$ and detection areas 


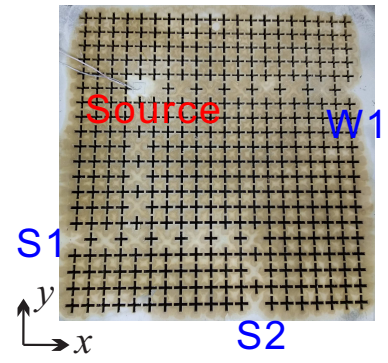

(a)

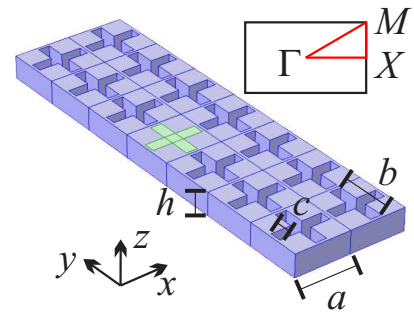

(b)
Figure 4: Coupled resonator elastic waveguides $(b / a=0.9$, $c / a=0.2, h / a=0.4$ ). (a) The experimental sample includes a linear chain of defect cavities defining a straight waveguide (W1), and a wave splitter circuit with $90^{\circ}$ bends and two output ports ( $\mathrm{S} 1$ and $\mathrm{S} 2$ ). The Lamb wave source is a piezoelectric patch. (b) The supercell of the coupled-resonator elastic waveguide is used to obtain the dispersion of guided waves.

$\left(S_{d}=a^{2}\right)$, i.e.

$$
T(f)=20 \log _{10}\left(\frac{\int_{S_{d}}\left|U_{z}\right| \mathrm{d} s}{\int_{S_{i}}\left|U_{z 0}\right| \mathrm{d} s}\right) .
$$

It should be noted that the transmission thus defined can be larger than one without violating energy conservation ${ }^{9}$.

\section{BANDGAPS OF THE PHONONIC CRYSTAL SLAB WITH CROSS HOLES}

We first consider a 2D square lattice PC slab with cross holes, as shown in Figure 2. The sample is composed of an array of $20 \times 20$ unit cells with a central defect where the source is attached. Actually, the consideration of cross holes is known to favor wide complete band gaps ${ }^{15}$. The geometrical parameters are chosen as $b / a=0.9, c / a=0.2$ and $h / a=0.4$, with the lattice constant $a=20 \mathrm{~mm}$, based on the optimization in Ref. ${ }^{15}$. The solid matrix is isotropic steel with mass density $\rho=7850 \mathrm{~kg} / \mathrm{m}^{3}$, Poisson's ratio $\nu=0.3$, and Young's modulus $E=206 \mathrm{GPa}$.

The phononic band structure of the perfect $\mathrm{PC}$ slab is shown in Fig. 3(a). The primitive unit cell and the corresponding first Brillouin zone are shown in Fig. 2(b). The dispersion bands are classified as in-plane or outof-plane but evaluating the displacement vector of each Bloch wave. Two complete bandgaps extend in the frequency ranges $53.15 \mathrm{kHz}<f<68.25 \mathrm{kHz}$ and $69.8 \mathrm{kHz}$ $<f<87.9 \mathrm{kHz}$. Experimental transmissions along the $\Gamma \mathrm{X}$ and $\Gamma \mathrm{M}$ directions are shown in Fig. 3(b). Excellent agreement is observed between numerical simulation and experiments, without any parametric adjustment. As a remark, the excitation is asymmetric with respect to the mid-plane of the slab and favors the excitation of out-of- plane modes. In-plane modes can, however, be excited as well as a result of the finite lateral extent of the source. The wide complete bandgaps are suitable for the design of highly confined coupled resonator waveguides, as we discuss next.

\section{COUPLED RESONATOR ELASTIC WAVEGUIDES}

In this section, we consider CREWs formed in the perfect PC slab by filling certain cross holes to form defect cavities. The sample fabricated in stainless steel is shown in Fig. 4(a). CREWs are formed by considering a chain of defect cavities separated by 2 lattice constants ${ }^{9}$. Both a straight waveguide and a wave splitter are fabricated on the same sample. The straight waveguide (W1) is a sequence of 7 cavities. The wave splitter is composed of one straight part and of two asymmetric parts (labeled $\mathrm{S} 1$ and S2), including sharp corners with $90^{\circ}$ bends ${ }^{7}$.

Experimental transmissions are shown in Fig. 5(a). They are normalized by the transmission for the straight waveguide measured at a low passing frequency. Three distinct guidance frequency ranges, labeled I-III, are clearly observed inside the complete bandgap. In the case of the wave splitter, the transmissions measured at the two ends present only slight differences, although their total lengths are quite different, similarly to the results in Ref. ${ }^{9}$. This observation is consistent with the high degree of confinement of guided waves. Experimental displacement fields at chosen frequencies for each of the three passing frequency ranges are presented in Figs. 5(b)-(d). Lamb waves are found to be strongly confined as they are guided along the waveguides and to turn efficiently at the $90^{\circ}$ bends. They are furthermore split rather evenly at the T-junction of the wave splitter. Away from the defect cavities, Lamb waves decay very fast in all directions. This is a further confirmation of the complete bandgap of the PC slab.

The phononic band structure shown in Fig. 6(a) is obtained considering a supercell of size $2 a \times 7 a \times h$, as depicted in Fig. 4(b). It indicates the existence of a total of 9 guided Bloch waves. Each of them emerges around a resonance frequency of the isolated defect cavity. The lowest 3 guided Bloch waves are mixed together and are thus left out of the following analysis, though they clearly lead to transmission both experimentally and theoretically. Eigenmodes at the $\mathrm{X}$ point are shown in Fig. 6(b). Guided Bloch waves 1, 5, and 6 are polarized mostly outof-plane. In contrast, guided Bloch waves 2, 3, and 4 are polarized mostly in-plane. The numerical transmissions through the waveguide and the wave splitter shown in Fig. 6(c) are in quite good agreement with the experimental results. Bloch waves 4 and 6 have a flat dispersion and thus can hardly be excited; hence they do not contribute to transmission. Since the wave source is vertical and symmetrical with respect to the $x z$ plane, longitudinal modes can be excited. The distribution of 

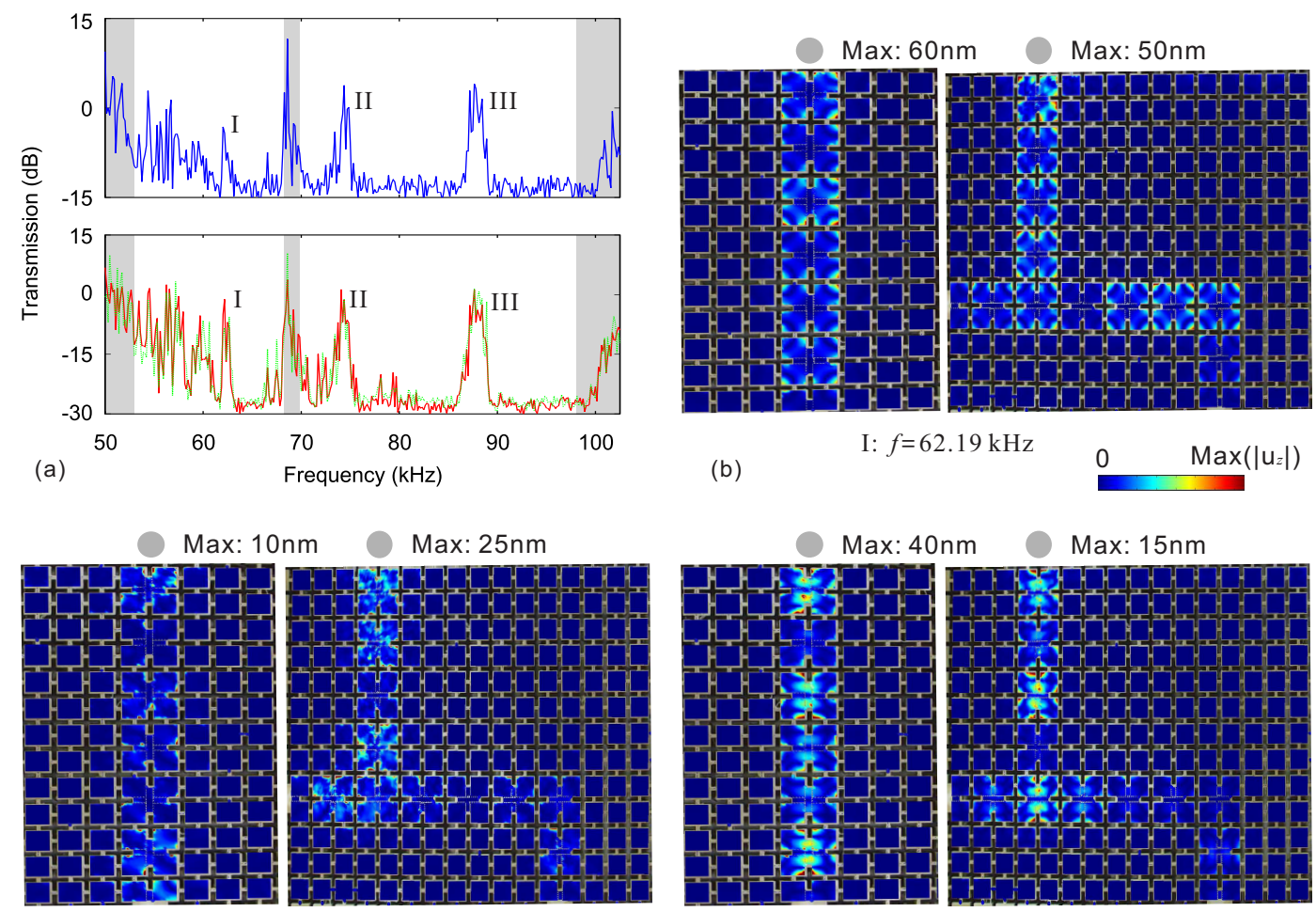

(c)

II: $f=73.44 \mathrm{kHz}$

(d)

III: $f=87.81 \mathrm{kHz}$

Figure 5: Experimental observation of Lamb wave propagation in the sample of Fig. 4. (a) The transmission through the waveguide (blue line: W1) and through the splitter (red line: S1, green line: S2) is shown as a function of frequency. Transmission is measured for the vertical component of displacement $\left|u_{z}\right|$ and is normalized to its value at low frequency. The gray areas indicate the passing bands for perfect PC slab. Panels (b)-(d) show field distributions of displacement measured at chosen frequencies of the three passing frequency ranges labeled I-III in (a). In each panel, the left and right subplots are for the straight waveguide or the wave splitter, respectively. The wave source position is indicated by the gray disk. The color scale is for amplitude of $z$-displacement normalized with respect to the maximum amplitude (Max).

$x$-displacement for Bloch wave 2 shown in Fig. $7(\mathrm{a})$ is symmetric with respect to the $x z$ plane. This mode can thus be excited, and results in a relatively high transmission. In contrast, the $x$-displacement distribution of Bloch wave 3 is asymmetric with respect to the $x z$ plane. Such a mode can then hardly be excited, and a quite low transmission is observed in both simulation and experiment. Summarizing, passing frequency ranges I, II and III can be attributed to Bloch waves 1,2 and 5, respectively. In each of these frequency ranges, guidance is single-mode. Taking range III for example, guiding and splitting of Lamb waves in Fig. 6(d) are clearly observed owing to the excitation of the eigenmode at X5 for Bloch wave 5 .

\section{INFLUENCE OF GEOMETRIC PARAMETERS}

The dispersion of Lamb waves is strongly dependent on the geometry ${ }^{15}$. Accordingly, we investigate the influence of geometric parameters, including slab thickness and size of the hole, on the dispersion of guided waves. The variation of the phononic band structure with the slab thickness is shown in Fig. 8. For a small thickness $(h / a=0.15)$, only passing bands appear below 94.5 $\mathrm{kHz}$. In the absence of a bandgap, guided modes cannot form between adjacent defect cavities. When $h / a=0.3$, guided modes are formed owing to the appearance of complete bandgaps. As the slab thickness is varied continuously, it is expected that bands shift continuously in dispersion space. Bands can then be labeled by comparing eigenmodes with those of Fig. 6(b). With $h / a$ increasing to 0.4 , the frequencies of Bloch waves 1,5 and 6 generally increase. Since these modes are mostly out-of-plane, their eigenfrequencies are to the first order proportional to the slab thickness. In contrast, the frequencies of Bloch waves 2, 3 and 4 do almost not change, since these modes are mostly in-plane. When $h / a=0.55$, Bloch waves 5 and 6 move to even higher frequencies, and Bloch wave 1 is mixed with the in-plane guided modes. When $h / a=0.9$, higher order vibration modes with cutoff frequencies ${ }^{15}$ appear, and are mixed with Bloch waves 3 and 4 . With a further increase of the slab thickness, these modes will close the bandgap ${ }^{15}$, causing the disappearance of the guided modes.

We further consider the effect of the hole length, $b / a$, on the guided modes. The results are illustrated in Fig. 

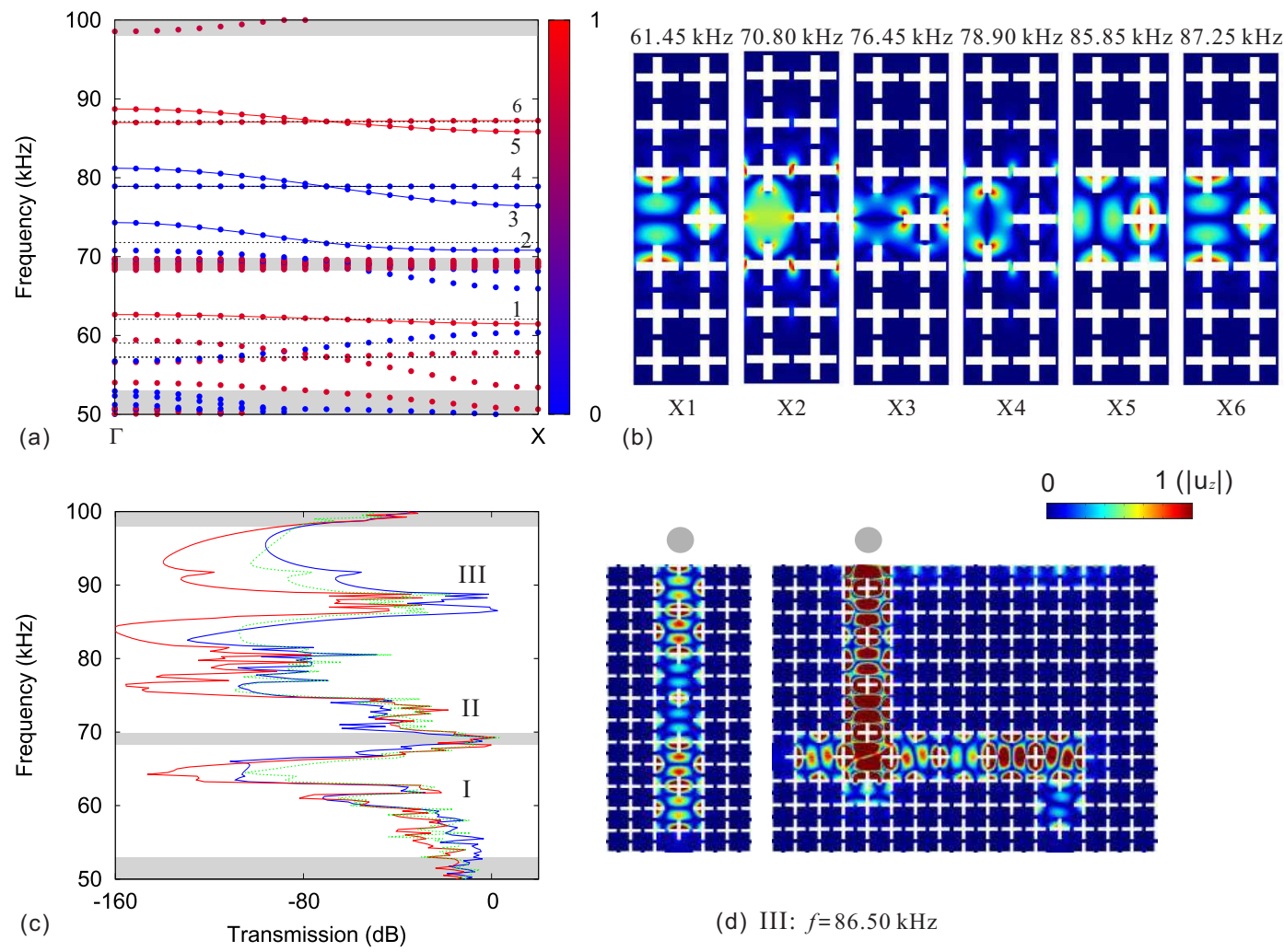

(d) III: $f=86.50 \mathrm{kHz}$

Figure 6: Finite element analysis of dispersion and transmission for coupled-resonator elastic waveguides of Fig. 5 . (a) The band structure is obtained from a supercell model of the periodic waveguide. Points are for finite element results. Solid lines are obtained by fitting to a theoretical model (see text). The horizontal dashed lines mark the resonant frequencies for an isolated cavity in the PC slab. The color scale represents from the in-plane modes (blue) to out-of-plane modes (red). The gray areas indicate the passing bands for the perfect PC slab. (b) The displacement distributions at the X point of the 6 Bloch waves labeled in (a) are shown. (c) The numerical transmission through the straight waveguide and the wave splitter is plotted as a function of frequency. Panel (d) shows the displacement distributions at a chosen frequency inside passing range III. The color scale in panels (b) and (d) is for amplitude of $z$-displacement normalized with respect to the maximum amplitude (Max).

Table I: Fitted expansion coefficients, $\Gamma_{m} /(2 \pi)(\mathrm{kHz})$, used for the prediction of CREW dispersion according to the theoretical model of Ref. ${ }^{8}$.

\begin{tabular}{llllllll}
\hline & Mode & $n=1$ & $n=2$ & $n=3$ & $n=4$ & $n=5$ & $n=6$ \\
\hline$h / a=0.3$ & $\Gamma_{0} /(2 \pi)$ & 54.04 & 72.12 & 78.78 & 78.83 & 75.38 & 75.27 \\
$b / a=0.9$ & $\Gamma_{1} /(2 \pi)$ & 0.37 & 0.88 & 1.19 & 0.005 & 0.81 & -0.09 \\
& $\Gamma_{2} /(2 \pi)$ & -0.02 & 0.21 & -0.019 & -0.001 & 0.054 & -0.001 \\
& $\Gamma_{3} /(2 \pi)$ & -0.002 & -0.06 & 0.003 & -0.0002 & 0.006 & -0.00009 \\
\hline$h / a=0.4$ & $\Gamma_{0} /(2 \pi)$ & 62.05 & 72.15 & 78.85 & 78.9 & 87.2 & 87.1 \\
$b / a=0.9$ & $\Gamma_{1} /(2 \pi)$ & 0.30 & 0.90 & 1.19 & 0.005 & 0.72 & -0.06 \\
& $\Gamma_{2} /(2 \pi)$ & -0.01 & 0.20 & -0.019 & -0.001 & 0.035 & -0.00048 \\
& $\Gamma_{3} /(2 \pi)$ & -0.001 & -0.06 & 0.004 & -0.0002 & 0.003 & -0.00003 \\
\hline$h / a=0.4$ & $\Gamma_{0} /(2 \pi)$ & 59.23 & 69.31 & 74.59 & 74.61 & 85.92 & 85.87 \\
$b / a=0.95$ & $\Gamma_{1} /(2 \pi)$ & 0.11 & 0.67 & 1.01 & -0.010 & 0.44 & -0.02 \\
& $\Gamma_{2} /(2 \pi)$ & -0.0008 & 0.08 & -0.031 & -0.002 & 0.015 & -0.00012 \\
& $\Gamma_{3} /(2 \pi)$ & -0.000 & -0.002 & 0.003 & -0.0003 & 0.0008 & -0.0000 \\
\hline
\end{tabular}

9. When $b / a=0.75$, a narrow complete bandgap exist in the considered frequency range and guided modes are mixed with passing bands. This mixture disappears when $b / a=0.8$, but the in-plane and the out-of-plane guided modes remain mixed. With a further increase of the hole length, Bloch waves 3 and 4 separate from Bloch waves
5 and 6 . When $b / a=0.9$, Bloch waves 1 and 2 come up. When $b / a=0.95$, Bloch wave 2 interferes with the passing bands around $70 \mathrm{kHz}$. As a rule, the frequencies of guided modes generally decrease with an increase in the hole length, similarly to the variation of bandgap edges reported in Ref. ${ }^{15}$. Furthermore, the frequencies 


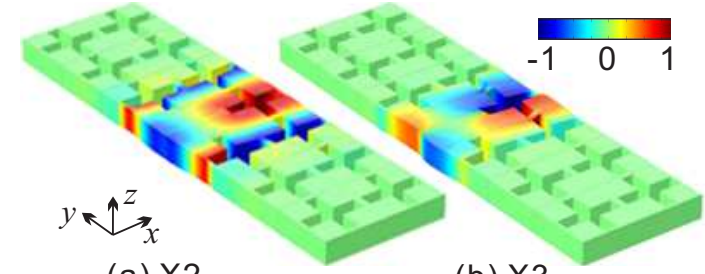

(a) $\times 2$

(b) $\times 3$

Figure 7: Distribution of the $x$-displacement at the $\mathrm{X}$ point for Bloch waves 2 and 3 in Fig. 6(a). The color scale varies from negative (blue) positive (red) normalized values.

of in-plane Bloch waves decrease faster than those of the out-of-plane Bloch waves.

The dispersion of guided Bloch waves in a linear chain of coupled cavities can be fitted against the theoretical model of Ref. ${ }^{8}$. The fitting parameters are listed in Table I for the six Bloch waves and for different geometrical parameters. As a note, parameter $\Gamma_{0} /(2 \pi)$ is the resonance frequency of the isolated cavity. The predicted dispersion relations are further shown in Figs. 6, 8 and 9 with solid lines. It can be seen that the fit of dispersion with the numerical results is excellent. Significantly, it is observed that the coupling coefficients hardly change with the slab thickness for the in-plane Bloch waves 2, 3 and 4. This is a direct evidence that the dispersion of the inplane guided Bloch waves is almost independent of the slab thickness. In contrast, coupling coefficients clearly vary with the hole length, especially for in-plane Bloch waves.

\section{CONCLUSIONS}

In summary, we have investigated coupled resonator elastic waveguides designed in a phononic crystal slab with cross holes. We first measured wide complete bandgaps using a finite PC slab sample. Coupledresonator elastic waveguides formed with chains of coupled cavities were then designed and fabricated. We observed the transmission of strongly confined Lamb waves along a straight waveguide and in a wave splitter circuit. Guidance and splitting of Lamb waves at $90^{\circ}$ bends were clearly observed in the experiments. Numerical simulations successfully explained experimental transmission results and allowed us to identify the guided Bloch waves responsible for guidance. The slab thickness was found to have little influence on the dispersion of in-plane guided Bloch waves, contrary to the usual case of Lamb waves of an homogeneous plate. The results in this paper can be used for the practical design of elastic wave devices based on coupled defect cavities at ultrasonic frequencies, and could be used to design more complicated phononic circuits.

\section{ACKNOWLEDGMENTS}

The authors acknowledge financial support from the National Natural Science Foundation of China (11702017 and 11532001), the Fundamental Research Funds for the Central Universities (2018JBM033), and the Labex ACTION program (Contract No. ANR-11-LABX-0001-01).
* Electronic address: wangyanfeng@bjtu.edu.cn

$\dagger$ Electronic address: yswang@bjtu.edu.cn

1 M. S. Kushwaha, P. Halevi, L. Dobrzynski, and B. DjafariRouhani, Physical Review Letters 71, 2022 (1993).

2 M. I. Hussein, M. J. Leamy, and M. Ruzzene, Applied Mechanics Reviews 66, 040802 (2014).

3 V. Laude, Phononic Crystals: Artificial Crystals for Sonic, Acoustic, and Elastic Waves (Walter de Gruyter GmbH, Berlin, 2015).

${ }^{4}$ X.-Z. Zhou, Y.-S. Wang, and C. Zhang, Journal of Applied Physics 106, 014903 (2009).

5 Y.-F. Wang, Y.-S. Wang, and X.-X. Su, Journal of Applied Physics 110, 113520 (2011).

6 M. Torres, F. R. Montero de Espinosa, D. Garcia-Pablos, and N. Garcia, Physical Review Letters 82, 3054 (1999).

7 A. Khelif, A. Choujaa, S. Benchabane, B. Djafari-Rouhani, and V. Laude, Applied Physics Letters 84, 4400 (2004).

8 J. M. Escalante, A. Martinez, and V. Laude, Journal of Physics D: Applied Physics 46, 475301 (2013).

\section{REFERENCES}

${ }^{9}$ Y.-F. Wang, T.-T. Wang, Y.-S. Wang, and V. Laude, Physical Review Applied 8, 014006 (2017).

10 T.-T. Wu, J.-C. Hsu, and J.-H. Sun, IEEE Transcations on Ultrasonics, Ferroelectrics, and Frequency Control 58, 2146 (2011).

11 A. Climente, D. Torrent, and J. Sanchez-Dehesa, Applied Physics Letters 105, 064101 (2014).

12 M. Miniaci, A. Marzani, N. Testoni, and L. D. Marchi, Ultrasonics 56, 251 (2015).

13 T.-T. Wang, Y.-F. Wang, Y.-S. Wang, and V. Laude, Applied Physics Letters 111, 041906 (2017).

14 B. Khelif, A.and Aoubiza, S. Mohammadi, A. Adibi, and V. Laude, Physical Review E 74, 046610 (2006).

15 Y.-F. Wang and Y.-S. Wang, Journal of Sound and Vibration 332, 2019 (2013).

16 F.-L. Hsiao, A. Khelif, H. Moubchir, A. Choujaa, C.-C. Chen, and V. Laude, Physical Review E 76, 056601 (2007).

17 M. Oudich, M. B. Assouar, and Z.-L. Hou, Applied Physics Letters 97, 193503 (2010). 


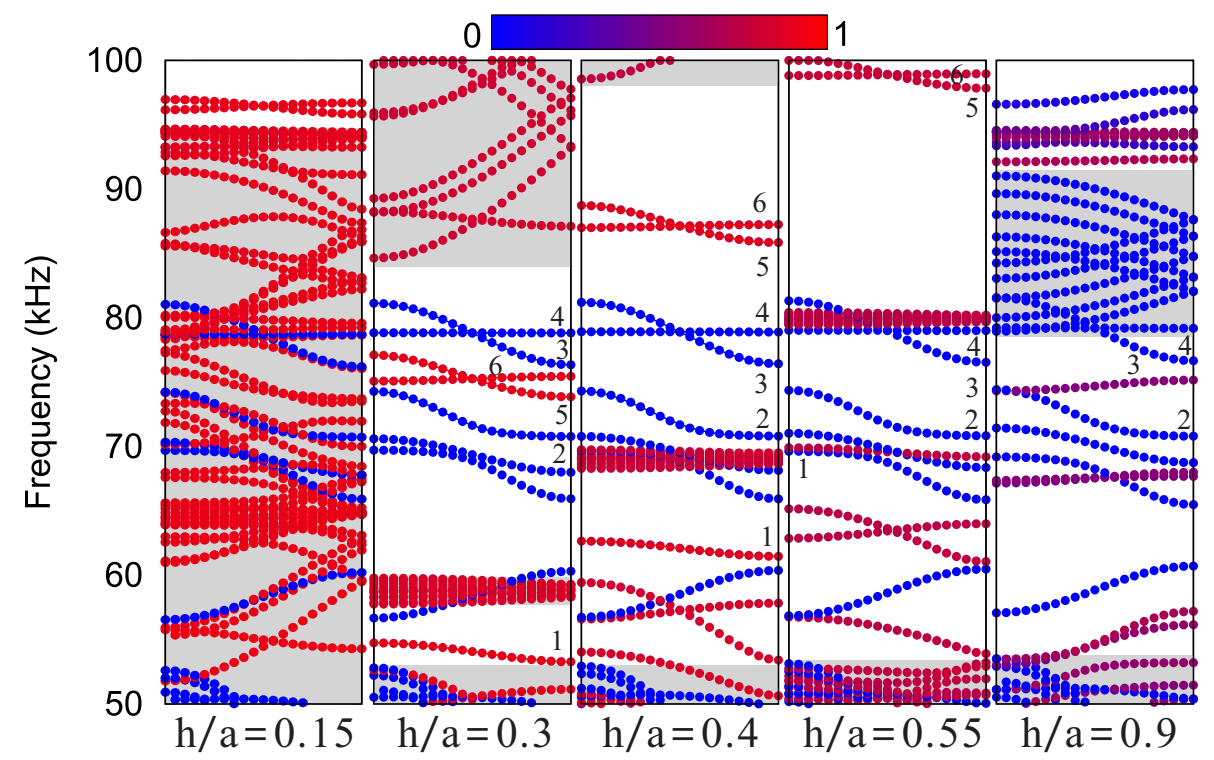

Figure 8: Band structures of CREWs with different relative thicknesses $(b / a=0.9, c / a=0.2)$. The color scale measures the polarization and varies from in-plane modes (blue) to out-of-plane modes (red). The gray areas indicate the passing bands for the corresponding perfect PC slab.

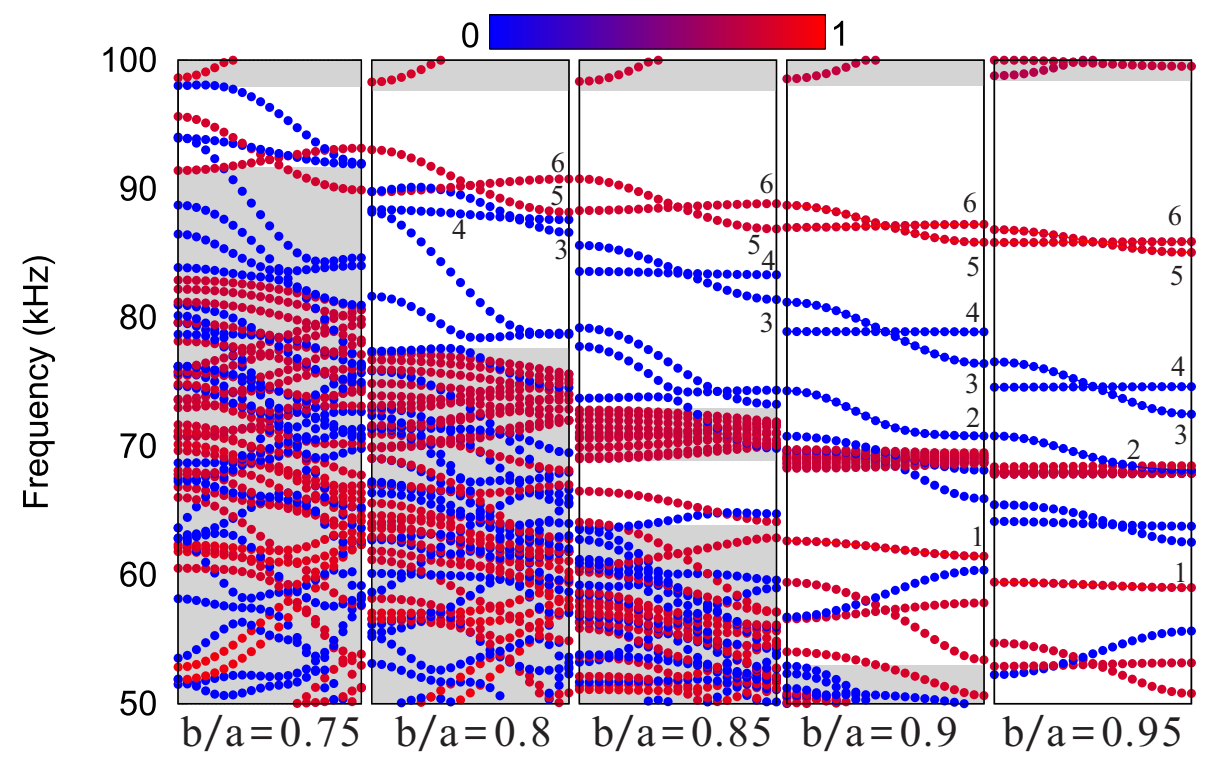

Figure 9: Band structures of CREWs with different relative hole lengths $(c / a=0.2, h / a=0.4)$. The color scale measures the polarization and varies from in-plane modes (blue) to out-of-plane modes (red). The gray areas indicate the passing bands for the corresponding perfect PC slab.

18 Y. Xiao, J.-H. Wen, and X.-S. Wen, Journal of Physics D: Applied Physics 45, 195401 (2012).

19 B. Bonello, C. Charles, and F. Ganot, Applied Physics Letters 90, 021909 (2007).

20 S. Mohammadi, A. A. Eftekhar, A. Khelif, W. D. Hunt, and A. Adibi, Applied Physics Letters 92, 221905 (2008).

${ }^{21}$ M. Gorisse, S. Benchabane, G. Teissier, C. Billard, A. Reinhardt, V. Laude, E. Defaÿ, and M. Aïd, Applied Physics Letters 98, 234103 (2011).

${ }^{22}$ C. M. Reinke, M. F. Su, R. H. Olsson, and I. EI-Kady, Applied Physics Letters 98, 061912 (2011).
${ }^{23}$ L. D'Alessandro, B. Bahr, L. Daniel, D. Weinstein, and R. Ardito, Journal of Computational Physics 344, 465 (2017).

${ }^{24}$ O. R. Bilal and M. I. Hussein, Applied Physics Letters 103, 111901 (2013).

25 M. B. Assouar and M. Oudich, Applied Physical Letters 100, 123506 (2012).

26 J.-H. Sun and T.-T. Wu, Physical Review E 76, 104304 (2007).

27 T.-C. Wu, T.-T. Wu, and J.-C. Hsu, Physical Review B 79, 104306 (2009). 
28 M. Ghasemi Baboly, A. Raza, J. Brady, C. M. Reinke, Z. C. Leseman, and E.-K. I., Applied Physics Letters 109, 183504 (2016).

${ }^{29}$ L. Mallet, B. C. Lee, W. J. Staszewski, and F. Scarpa,
Smart Materials and Structures 13, 261 (2004).

30 W. H. Leong, W. J. Staszewski, B. C. Lee, and F. Scarpa, Smart Materials and Structures 14, 1387 (2005). 